\title{
Multisegment Foot Kinematics During Walking in Younger and Older Adults
}

\author{
Dominique Legault-Moore ${ }^{\mathrm{a}}$, Victoria L. Chester ${ }^{\mathrm{a}, \mathrm{c}}$, Gwyneth de Vries ${ }^{\mathrm{b}}$
}

\begin{abstract}
Background: Currently, age-related changes in foot mechanics are poorly understood. A greater understanding of the natural changes in foot motion is needed to improve our understanding of pathological foot conditions.
\end{abstract}

Methods: The purpose of this study was to compare multisegment foot kinematic data during gait in younger and older individuals. Eleven $(\mathrm{N}=11)$ adult male participants between the ages of $18-30$ years (younger group; mean $\pm \mathrm{SD}: 24.6 \pm 3.0$ years) and eleven $(\mathrm{N}=11)$ adults aged 55 years or older (older group; mean $\pm \mathrm{SD}$ : $65.0 \pm 4.2$ years) were recruited for the study. The foot was modeled as a four-segment rigid body model. Three-dimensional kinematic and kinetic gait parameters were recorded using an 8-camera Vicon MCam motion capture system and two Kistler force plates. A MANOVA was used to test for significant differences in mean temporal-spatial data, mean ranges of motion, and mean peak joint angle data between age groups.

Results: No significant differences $(P>0.05)$ were found between the two age groups for any of the gait parameters. The results of the present study suggest that individuals aged $65.0 \pm 4.2$ years have foot mechanics that are comparable to younger walkers.

Conclusions: As such, any deviations in motion at this age may be indicative of an underlying disease or disorder.

Keywords: Gait; Multisegment foot; Aging; Kinematics

\footnotetext{
Manuscript accepted for publication May 31, 2012

${ }^{a}$ Andrew and Marjorie McCain Human Performance Laboratory, Richard J. Currie Center, Faculty of Kinesiology, University of New Brunswick, Fredericton, NB, E3B 5A3, Canada

${ }^{\mathrm{b}}$ Department of Orthopaedic Surgery, Dr. Everett Chalmers Hospital, Fredericton, New Brunswick, E3B 6H5, Canada

${ }^{\mathrm{c} C}$ Corresponding author: Victoria L. Chester. Email: vchester@unb.ca
}

doi:10.4021/jocmr984w

\section{Introduction}

The foot plays a critical role during gait, providing a base of support and aiding in shock absorption and propulsion. To date, the majority of foot biomechanics research has modeled the foot as a single rigid body. Over the last decade, there has been an increase in the number of studies that have used multisegment foot models [1-4]. These models have increased our knowledge of the complex movements within the foot during gait. Such models are beneficial for research examining the relationship between foot function and aging, disease, and treatment efficacy.

Previous research has suggested that changes in foot structure and function occur with age. Changes include decreased range of joint motion [5-9], decreased strength [5, 8-9], lowering of the transverse arch [9-10], and decreased proprioception [11]. Such changes may lead to alterations in foot mechanics and could have important consequences to an individual's mobility and quality of life.

Gait studies that compared the foot biomechanics of healthy younger and older populations have typically used single rigid segment models. Decreased ankle plantarflexion [12-15], decreased ankle joint range of motion [16], reduced peak ankle joint power [14, 17-18], and a more flat-footed landing [14], have been reported for older walkers. However, research using more complex multisegment models of the foot in the aging population is limited. The majority of studies examining healthy feet using multisegment models have typically assessed children to adults [1-4, 19-24]. Studies that included individuals aged 55 years or older have combined the data for the older participants with younger participants $[19,23]$. Therefore, we currently know very litte about the multisegment foot mechanics in older adults specifically. Multisegment foot motion could provide additional insight into age-related changes in foot mechanics in older populations.

To our knowledge, no studies have compared multisegment foot kinematics in younger and older age groups. Research that examines multisegment foot kinematics across age spans is needed to increase our knowledge of age-related changes in foot movement. A greater understanding of these 
Table 1. Participant Characteristics (Mean \pm 1 SD) for the Older and Younger Groups

\begin{tabular}{lccccc}
\hline & \multicolumn{2}{c}{ Younger } & & \multicolumn{2}{c}{ Older } \\
\cline { 2 - 3 } \cline { 5 - 6 } & Mean & SD & & Mean & SD \\
\hline Age (years) & 24.6 & 3.0 & & 65.0 & 4.2 \\
Height (cm) & 178.5 & 5.8 & & 174.6 & 6.3 \\
Weight (kg) & 79.5 & 9.7 & & 78.7 & 14.6 \\
Foot Size & 10.3 & 1.1 & 9.8 & 1.1 \\
\hline
\end{tabular}

Table 2. Anatomical Landmarks for the Four-Segment Model of the Foot and Shank

\section{Segment Location}

Hallux Most distal and dorsal point of the head of the proximal phalanx

Forefoot Head of the fifth metatarsal, dorso-lateral aspect of the fifth metatarso-phalangeal joint

Head of the second metatarsal, dorso-medial aspect of the second metatarso-phalangeal joint

Head of the first metatarsal, dorso-medial aspect of the first metatarso-phalangeal joint

Base of the fifth metatarsal, dorso-lateral aspect of the fifth metatarso-cuboid joint

Base of the second metatarsal, dorso-medial aspect of the second metatarso-cuneiform joint

Base of the first metatarsal, dorso-medial aspect of the first metatarso-cuneiform joint

Calcaneus Lateral calcaneus - midpoint between the lateral malleolus and floor

Medial calcaneus - point between medial malleolus and floor at height of lateral calcaneus marker

Posterior calcaneus - aligned vertically with Achilles tendon at same height as lateral calcaneus marker

Shank Most lateral aspect of lateral malleolus

Most medial aspect of the medial malleolus

Most lateral prominence of lateral epicondyle

Most medial prominence of the medial epicondyle

Most anterior aspect of the tibial tuberosity 


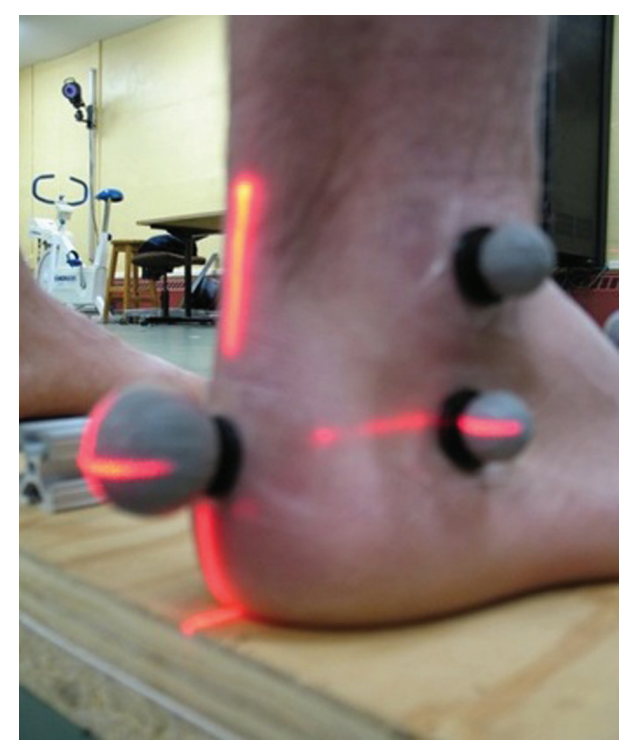

Figure 1. Laser technique used to align calcaneus markers. naturally occurring changes could play an important role in identifying foot/ankle pathology in older populations. Therefore, the purpose of this study is to compare multisegment foot kinematic data during gait in younger and older individuals.

\section{Method}

\section{Participants}

Twenty-two $(\mathrm{N}=22)$ adult male participants between the ages of 18-30 years (younger group; $\mathrm{N}=11$ ) and 55 years or older (older group; $\mathrm{N}=11$ ) were recruited for the study. Further characteristics of each group are provided in Table 1. With the exception of age, there were no significant differences $(\mathrm{P}>0.05)$ between the old and young groups for mass, height, or shoe size. All participants had a minimum shoe size of nine to facilitate marker tracking and autolabeling. Female participants were excluded from the study for two

Table 3. Descriptive Data (Mean \pm 1 S.D.) for Temporal-Spatial Variables for the Younger and Older Age Groups

\begin{tabular}{|c|c|c|c|c|}
\hline \multirow{2}{*}{$\begin{array}{l}\text { Temporal-spatial } \\
\text { Measure }\end{array}$} & \multicolumn{2}{|c|}{ Younger $(\mathrm{N}=11)$} & \multicolumn{2}{|c|}{ Older $(\mathrm{N}=11)$} \\
\hline & Mean & SD & Mean & SD \\
\hline Cycle Time (s) & 1.1 & 0.1 & 1.1 & 0.1 \\
\hline Cadence (steps/min) & 106.2 & 4.6 & 107.6 & 8.7 \\
\hline Stride Length (m) & 1.4 & 0.1 & 1.4 & 0.2 \\
\hline Walk Speed (m/s) & 1.2 & 0.1 & 1.2 & 0.2 \\
\hline Opposite Toe-off (\%) & 12 & 1.5 & 11.5 & 1.7 \\
\hline Opposite Foot Strike (\%) & 49.6 & 1.5 & 50.1 & 1.6 \\
\hline Toe-off $(\%)$ & 61.3 & 1.3 & 60.7 & 1.6 \\
\hline Single Stance (\%) & 37.6 & 1.2 & 38.6 & 2 \\
\hline Double Stance (\%) & 23.6 & 2.2 & 22 & 2.8 \\
\hline Stance $(\%)$ & 61.3 & 1.3 & 60.7 & 1.6 \\
\hline
\end{tabular}


Table 4. Descriptive Results (Mean \pm 1 S.D.) for Joint Angles for the Younger and Older Groups During the Stance Phase, Swing Phase, and Entire Gait Cycle

\begin{tabular}{|c|c|c|c|c|c|}
\hline \multirow{2}{*}{ Variables } & \multirow{2}{*}{ Phase } & \multicolumn{2}{|c|}{$\begin{array}{l}\text { Young Group } \\
\qquad(\mathrm{N}=11)\end{array}$} & \multicolumn{2}{|c|}{$\begin{array}{l}\text { Old Group } \\
\qquad(\mathrm{N}=11)\end{array}$} \\
\hline & & Mean $\left({ }^{\circ}\right)$ & SD & $\operatorname{Mean}\left({ }^{\circ}\right)$ & SD \\
\hline \multicolumn{6}{|l|}{ Hindfoot - Shank } \\
\hline Max inversion & Cycle & -8.00 & 5.20 & -7.40 & 3.20 \\
\hline Max eversion & Cycle & 4.50 & 3.00 & 4.70 & 1.80 \\
\hline Eversion ROM & Cycle & 12.50 & 3.50 & 12.10 & 3.00 \\
\hline Max plantarflexion & Stance & -11.30 & 2.20 & -12.20 & 3.90 \\
\hline Max plantarflexion & Swing & -14.80 & 6.40 & -12.20 & 8.00 \\
\hline Max dorsiflexion & Cycle & 8.50 & 2.40 & 9.20 & 3.30 \\
\hline Dorsiflexion ROM & Cycle & 19.80 & 2.10 & 21.40 & 2.40 \\
\hline Max abduction & Cycle & -10.30 & 2.60 & -9.80 & 4.20 \\
\hline Max abduction & Cycle & 3.80 & 2.70 & 5.00 & 2.10 \\
\hline Abduction ROM & Cycle & 14.10 & 2.90 & 14.70 & 3.60 \\
\hline \multicolumn{6}{|l|}{ Hindfoot - Forefoot } \\
\hline Max eversion & Cycle & 8.40 & 3.50 & 9.10 & 2.00 \\
\hline Max plantarflexion & Cycle & -13.50 & 3.90 & -11.70 & 4.10 \\
\hline Max dorsiflexion & Cycle & 3.70 & 3.40 & 4.40 & 3.10 \\
\hline Dorsiflexion ROM & Cycle & 17.20 & 5.60 & 16.10 & 3.80 \\
\hline \multicolumn{6}{|l|}{ Foot - Shank } \\
\hline Max inversion & Cycle & -8.80 & 3.30 & -12.00 & 4.20 \\
\hline Max plantarflexion & Stance & -12.40 & 5.10 & -10.60 & 5.00 \\
\hline Max plantarflexion & Swing & -17.40 & 7.30 & -14.60 & 4.30 \\
\hline Max dorsiflexion & Cycle & 12.30 & 2.30 & 12.40 & 4.50 \\
\hline Dorsiflexion ROM & Cycle & 24.70 & 4.60 & 23.00 & 3.60 \\
\hline \multicolumn{6}{|l|}{ Planar Angles [1] } \\
\hline V2G Max & Cycle & 74.10 & 11.80 & 68.30 & 12.50 \\
\hline S2G Max & Cycle & 107.40 & 10.00 & 102.60 & 10.90 \\
\hline F2Ps Max & Cycle & 45.50 & 3.80 & 41.80 & 8.60 \\
\hline MLA Max & Cycle & 171.50 & 7.30 & 171.20 & 6.90 \\
\hline
\end{tabular}



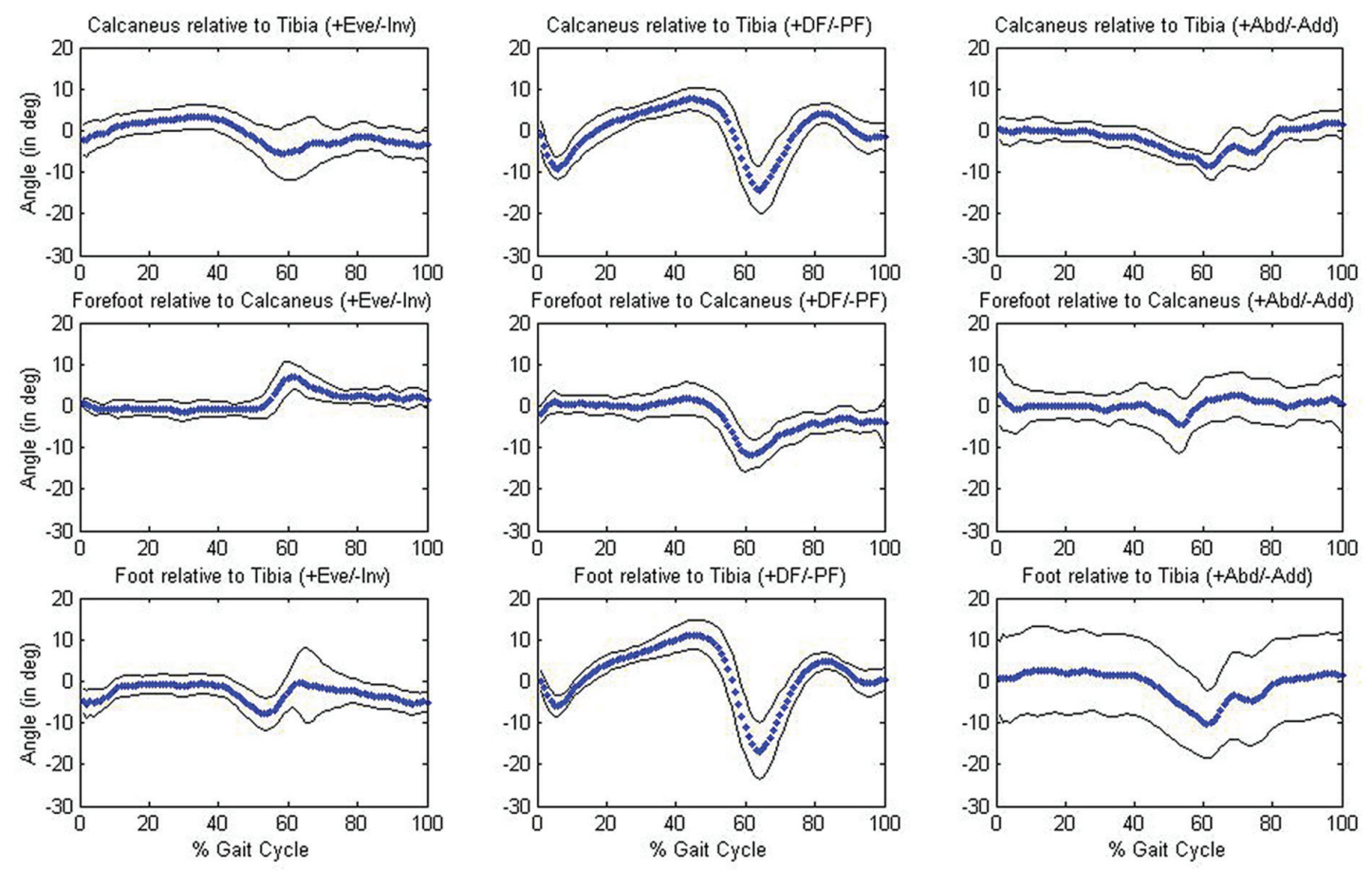

Figure 2. Joint angles for the younger group. Graphs in the first row of figure depict the calcaneus relative to the tibia; graphs in the second row depict the forefoot relative to the calcaneus; graphs in the third row depict the whole foot relative to the tibia.

reasons: 1) on average, females have smaller feet than males, which causes an increase in the incidence of marker merging and tracking problems; and 2) a sample of only males eliminates any gender effects that may be associated with foot kinematics. A medical history questionnaire was completed by each individual. Individuals with a history of diseases/ disorders that could affect foot kinematics, including diabetes, neurological pathologies, joint replacement surgeries, chronic pain, and edema were excluded from the study. Participants were recruited through advertisements, emails, and word-of-mouth. This study was approved by the University Research Ethics Board.

\section{Instrumentation}

An eight camera Vicon MCam motion capture system (Oxford Metrics Group, Oxford, UK), sampling at $120 \mathrm{~Hz}$, was used to track the three-dimensional trajectories of reflective markers (diameter of 14 and $25 \mathrm{~mm}$ ) placed on the participant's skin. Two force plates (Kistler 9281CA, Kistler Instruments, Winterthur, Switzerland), embedded and disguised in the lab floor, were used to aid in the identification of gait cycle events. A weight scale, measuring tape, and calipers were used to obtain anthropometric measures from each subject.

\section{Procedures}

Fifteen $(\mathrm{N}=15)$ reflective markers were placed directly on the right foot and tibia of each participant (Table 2). For consistency, the same researcher was always responsible for placing markers on each participant in the study. Following this, a static capture of the participant during quiet standing in the anatomical position was recorded to permit the calculation of offset values for all joint rotations. These joint offset values were later subtracted from the appropriate joint rotations for the gait cycles of each participant. This offset calculation did not apply to the planar angles. Following the static trial, each participant was asked to walk barefoot across the lab at a self-selected speed. Several practice trials were completed to allow the participants to adjust to the markers and the lab environment. Participants were then asked to perform at least 6 successful right limb gait cycles (e.g. clean force plate strike and marker visibility) at a selfselected speed.

\section{Multisegment foot model}

Four foot segments were created and assumed to be rigid: 1) the shank; 2) the total foot (single rigid segment); 3 ) the calcaneus; and 4) the forefoot (included all 5 metatarsal bones). The hallux and metatarsal bones were modeled as line segments for the computation of planar angles. The anatomical landmarks (Table 2) and reference frames were consistent with the model developed by Leardini et al [1], with the following exceptions: 1) the midfoot segment was excluded from the present study due to difficulties with con- 

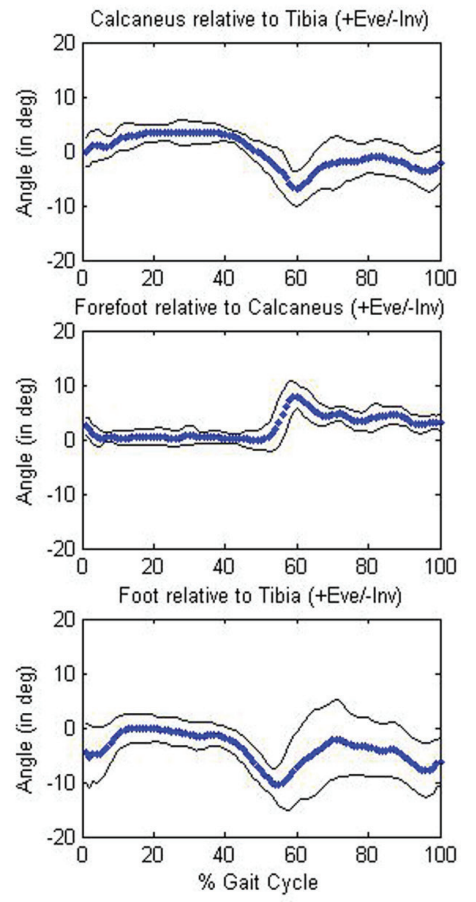
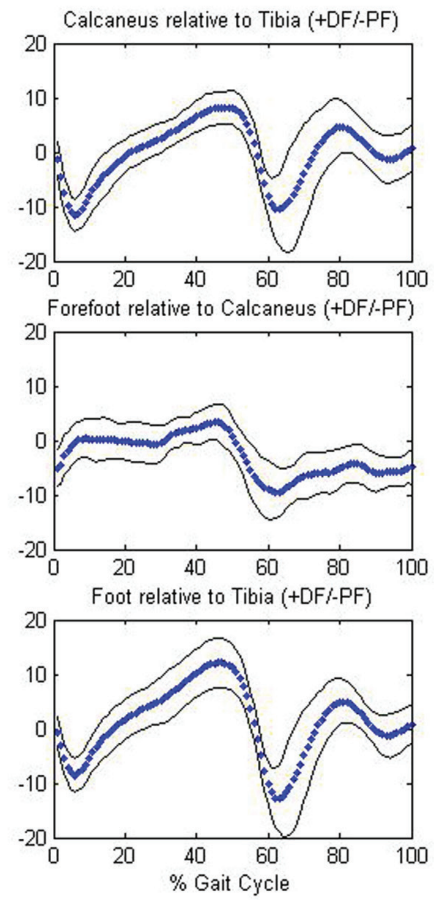
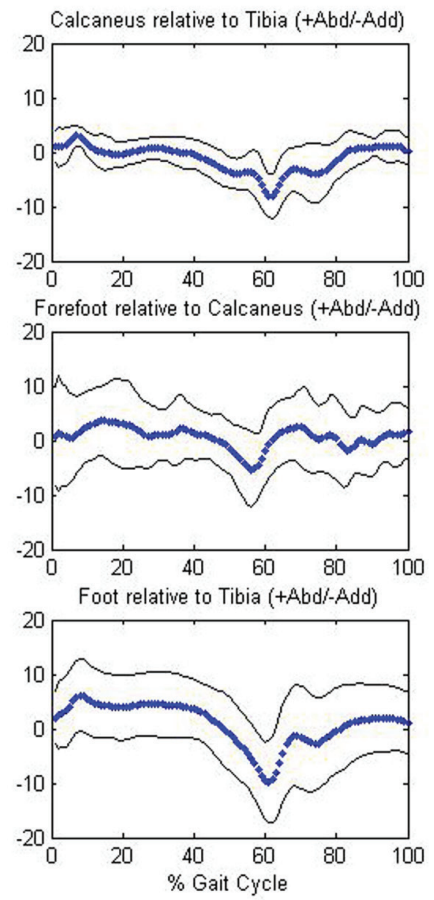

Figure 3. Joint angles for the older group. Graphs in the first row of figure depict the calcaneus relative to the tibia; graphs in the second row depict the forefoot relative to the calcaneus; graphs in the third row depict the whole foot relative to the tibia.

sistent tracking of the navicular and cuboid markers; and 2) in contrast to Leardini et al [1], a neutral calcaneus was formed using a laser level technique to guide marker placement (Fig. 1). This ensured the reliable placement of markers on a segment with few palpable anatomical landmarks. To achieve a neutral calcaneus, the vertical midpoint between the floor and the lateral malleolus was determined using calipers. A marker was placed at this point and referred to as the lateral calcaneus. A cross-hair laser was then used to aid in the placement of the posterior calcaneus marker. The vertical laser line was aligned with the midsection of the Achilles tendon, while the horizontal laser line was aligned with the lateral calcaneus marker. The intersection of the two laser lines formed the location for the posterior calcaneus marker. Lastly, using the laser level, the medial calcaneus marker was placed beneath the medial malleolus at the same height as the lateral and posterior calcaneus markers.

\section{Data analysis}

Data was analyzed using custom software created in Matlab (Mathworks, Inc). For each participant, trial selection involved the computation of cadence, velocity, and percent of cycle spent in single stance for each successful gait cycle. The single gait cycle that most closely approximated the individual mean of all gait cycles on these three measures was selected as the single trial for analysis for each participant. The locations of the three non-collinear markers on each rigid segment were used to create embedded coordinate systems at the virtual joint centers [1]. Joint angles were computed from the relative orientations of the embedded coordinate systems using Euler angles in an yxz sequence, corresponding to flexion/extension, adduction/abduction, and internal/external rotation. Displacement data were filtered using a zero phase lag, second order Butterworth filter with a cutoff frequency of $6 \mathrm{~Hz}$. Joint angle data were normalized to $100 \%$ of the gait cycle. A MANOVA was used to test for significant differences in mean temporal-spatial data (Table 3 ), mean range of motion, and mean peak joint angle data between age groups (Table 4).

\section{Results}

Significant $(\mathrm{P}<0.05)$ differences in kinematic parameters between the younger and older age groups were tested using a MANOVA. No significant differences $(\mathrm{P}>0.05)$ in mean temporal-spatial or mean joint angle parameters were found between age groups. Descriptive data for the temporalspatial and joint angle data for each group are provided in Tables 3-4. Overall, mean temporal-spatial and mean joint angle parameters were very similar across the two groups. Of particular importance was that mean walking speed was very comparable in the young $(1.20 \mathrm{~m} / \mathrm{s})$ and old $(1.22 \mathrm{~m} / \mathrm{s})$ groups. The older group showed slightly larger variability across variables. Figure 2 and Figure 3 provide graphical re- 
sults of the joint angles examined.

\section{Discussion}

The present study examined age-related differences in multisegment foot kinematics during gait. No significant differences $(P>0.05)$ were found between the younger and older age groups for the mean temporal-spatial or mean joint angle data. In addition, no significant differences $(\mathrm{P}>0.05)$ were found in height, mass, and shoe size measures between groups. As previous studies have not examined age-related changes in multisegment foot kinematics, comparisons of data across studies were not possible. However, for both the younger and older group, multisegment foot kinematic data was similar to previously published control data from numerous studies [1, 20-26].

Previous gait studies using single rigid segment models of the foot have reported varying results for ankle angles as a function of age. For example, Judge et al [13] found decreased plantarflexion in late stance in a group of older walkers (mean age: 79 years; range: 70 - 90 years) compared to younger walkers (mean age: 26 years; range: 18 - 42 years). The more advanced age of the older walkers in Judge et al [13] versus the present study may account for the differential results for the ankle joint. However, DeVita and Hortobagyi [27] also found decreased plantarflexion for older walkers (mean \pm SD: $69 \pm 6.5$ years) compared to younger participants (mean \pm SD: $21.6 \pm 2.7$ years) who were similar in age to the present study. In contrast, Ostrosky and vanSwearingen [28] found no significant differences in ankle plantarflexion between older (mean: 67.4 years; range: 60 - 80 years) and younger (mean: 28.2 years; range: 22 - 39 years) walkers.

Previous research has also reported decreased ankle range of motion during walking in older walkers versus younger walkers. Hageman and Blanke [16] reported decreased ankle range of motion in older women (mean: 66.85 years; range: 60.0 - 84.0 years) compared to younger women (mean: 23.92 years; range: 20.0 - 33.0 years) while walking at a self-selected speed. These differences were hypothesized to be related to the slower walking velocity of the older group. However, Kerrigan et al [18] reported decreased plantarflexion angles in the elderly (mean: 72.7 years; range: $65-84$ years) at walking speeds that were both faster and slower than the comfortable walking speed of a young control group (mean: 28.5 years; range: 18 - 36 years). In the present study, mean self-selected walking velocities were similar for the younger and older groups and no significant differences were found in ankle range of motion.

Similar foot mechanics between age groups has important implications for clinicians assessing foot pathology in older adults. Research to date has suggested that foot function changes with age. The results of the present study sug- gest that individuals aged $65.0 \pm 4.2$ years have typical foot mechanics during gait. Therefore, deviations in motion at this age may be indicative of an underlying disease or disorder.

A limitation of this study includes the small sample size of both the younger and older age groups $(\mathrm{N}=11$ for each group). Additionally, while individuals aged 55 years or older were sought for this study, the recruited older participants formed a group with a narrow age range. Therefore, a crosssection of the elderly population was not achieved. The results of this study are specific to the age range and gender of the individuals that participated.

\section{Conclusions}

This is the first study to provide a comparison of younger and older multisegment foot kinematics during gait. A greater understanding of the natural changes in foot mechanics with aging will allow clinicians to more accurately identify foot pathology. The lack of significant differences between the two age groups suggests that normal foot mechanics are present in older individuals. Abnormal foot mechanics as a function of age should not be assumed. Future work will focus on repeating the study with larger sample sizes to validate the findings. In addition, female participants and samples with wider age ranges will be included.

\section{References}

1. Leardini A, Benedetti MG, Berti L, Bettinelli D, Nativo R, Giannini S. Rear-foot, mid-foot and fore-foot motion during the stance phase of gait. Gait Posture. 2007;25(3):453-462.

2. Nester C, Jones RK, Liu A, Howard D, Lundberg A, Arndt A, Lundgren P, et al. Foot kinematics during walking measured using bone and surface mounted markers. J Biomech. 2007;40(15):3412-3423.

3. Simon J, Doederlein L, McIntosh AS, Metaxiotis D, Bock HG, Wolf SI. The Heidelberg foot measurement method: development, description and assessment. Gait Posture. 2006;23(4):411-424.

4. Tulchin K, Orendurff M, Karol L. A comparison of multisegment foot kinematics during level overground and treadmill walking. Gait Posture. 2010;31(1):104-108.

5. Edelstein JE. Foot care for the aging. Phys Ther. 1988;68(12):1882-1886.

6. James B, Parker AW. Active and passive mobility of lower limb joints in elderly men and women. Am J Phys Med Rehabil. 1989;68(4):162-167.

7. Nigg BM, Fisher V, Allinger TL, Ronsky JR, Engsberg JR. Range of motion of the foot as a function of age. Foot Ankle. 1992;13(6):336-343.

8. Endo M, Ashton-Miller JA, Alexander NB. Effects of 
age and gender on toe flexor muscle strength. J Gerontol A Biol Sci Med Sci. 2002;57(6):M392-397.

9. Scott G, Menz HB, Newcombe L. Age-related differences in foot structure and function. Gait Posture. 2007;26(1):68-75.

10. Hsu JD. Foot problems in the elderly patient. J Am Geriatr Soc. 1971;19(10):880-886.

11. Stevens JC, Choo KK. Spatial acuity of the body surface over the life span. Somatosens Mot Res. 1996;13(2):153166.

12. Murray MP, Kory RC, Sepic SB. Walking patterns of normal women. Arch Phys Med Rehabil. 1970;51(11):637650.

13. Judge JO, Davis RB, 3rd, Ounpuu S. Step length reductions in advanced age: the role of ankle and hip kinetics. J Gerontol A Biol Sci Med Sci. 1996;51(6):M303-312.

14. Winter DA, Patla AE, Frank JS, Walt SE. Biomechanical walking pattern changes in the fit and healthy elderly. Phys Ther. 1990;70(6):340-347.

15. Kaneko M, Morimoto Y, Kimura M, Fuchimoto K, Fuchimoto T. A kinematic analysis of walking and physical fitness testing in elderly women. Can J Sport Sci. 1991;16(3):223-228.

16. Blanke DJ, Hageman PA. Comparison of gait of young men and elderly men. Phys Ther. 1989;69(2):144-148.

17. Crowinshield RD, Brand RA, Johnston RC. The effects of walking velocity and age on hip kinematics and kinetics. Clin Orthop Relat Res. 1978132):140-144.

18. Kerrigan DC, Todd MK, Della Croce U, Lipsitz LA, Collins JJ. Biomechanical gait alterations independent of speed in the healthy elderly: evidence for specific limiting impairments. Arch Phys Med Rehabil. 1998;79(3):317-322.

19. Canseco K, Long J, Marks R, Khazzam M, Harris G. Quantitative characterization of gait kinematics in patients with hallux rigidus using the milwaukee foot mod- el. Journal of Orthopaedic Research. 2007;26(4):419427.

20. Carson MC, Harrington ME, Thompson N, O'Connor JJ, Theologis TN. Kinematic analysis of a multi-segment foot model for research and clinical applications: a repeatability analysis. J Biomech. 2001;34(10):12991307.

21. Jenkyn TR, Nicol AC. A multi-segment kinematic model of the foot with a novel definition of forefoot motion for use in clinical gait analysis during walking. J Biomech. 2007;40(14):3271-3278.

22. Kidder SM, Abuzzahab FS, Jr., Harris GF, Johnson JE. A system for the analysis of foot and ankle kinematics during gait. IEEE Trans Rehabil Eng. 1996;4(1):25-32.

23. Kitaoka HB, Crevoisier XM, Hansen D, Katajarvi B, Harbst K, Kaufman KR. Foot and ankle kinematics and ground reaction forces during ambulation. Foot Ankle Int. 2006;27(10):808-813.

24. Leardini A, Benedetti MG, Catani F, Simoncini L, Giannini S. An anatomically based protocol for the description of foot segment kinematics during gait. Clin Biomech (Bristol, Avon). 1999;14(8):528-536.

25. Tulchin K, Karol L. Multi-segment foot motion in healthy individuals: Comparsion of children and adults. Gait \& Posture. 2006;24S:S219-S220.

26. Myers KA, Wang M, Marks RM, Harris GF. Validation of a multisegment foot and ankle kinematic model for pediatric gait. IEEE Trans Neural Syst Rehabil Eng. 2004;12(1):122-130.

27. DeVita P, Hortobagyi T. Age causes a redistribution of joint torques and powers during gait. J Appl Physiol. 2000;88(5):1804-1811.

28. Ostrosky KM, VanSwearingen JM, Burdett RG, Gee Z. A comparison of gait characteristics in young and old subjects. Phys Ther. 1994;74(7):637-644; discussion 644-636. 